\title{
Semiflexible Rod in a Nematic Environment with Applications to Biological Polymers
}

\author{
D. R. Daniels* and M. S. Turner \\ Department of Physics, University of Warwick, Coventry CV4 7AL, UK \\ Received March 9, 2004; Revised Manuscript Received October 18, 2004
}

\begin{abstract}
We present the theory for a long polymer rod immersed in a nematic environment and find the effects of a nematic solvent on small fluctuations of the tangent vector perpendicular to the rod axis. We are thus able to calculate the physically interesting properties associated with our combined polymer and nematic system. These include tangent-tangent correlation functions and the scattering structure factor, which reproduces known qualitative experimental results rather well. As one biological application of our model, we analyze the possible effects of a weak nematic solvent on the mechanical properties of individual hemoglobin fibers. As another biological application, we model the effects of a strong nematic environment, provided by fd virus, on the elastic and conformational properties of wormlike micelles. The work presented here can be viewed as constituting a microscopic model derivation of previous, more phenomenologically inspired, theories that deal with similar polymer/nematic systems. Interestingly, we find that a simple boundary condition of the nematic order at the rod surface agrees well with available experimental data.
\end{abstract}

\section{Introduction}

It is of general physical interest in soft condensed matter, and often of considerable biological interest, to take into account the effects of a nematic environment on the conformational, elastic, and mechanical properties of single polymer chains. ${ }^{1-3}$ Many biological macromolecules, such as DNA, ${ }^{4-6}$ proteins, ${ }^{7-9}$ and TMV,${ }^{10-12}$ are long, semiflexible, slender rods where the role of nematic order can be important. In this paper we present a theoretical model for a single semiflexible polymer rod immersed in a nematic solvent. Our model differs from some previous theoretical approaches to this topic, ${ }^{13-18}$ in that we do not posit a phenomenologically inspired (albeit well motivated) potential of nematic origin for our polymer chain from the outset. Rather, we derive an effective potential for tangential fluctuations of our polymer rod by "integrating out" the degrees of freedom associated with the nematic field, subject to the constraint that (at the rod's surface) the rod tangent vector must align on average with that of the nematic director. The nematic field is taken to be governed by the usual Frank elasticity free energy. ${ }^{1-3}$ In section 2 we outline the theory used and find the effect of a nematic solvent on tangential rod fluctuations perpendicular to the long axis of the rod. To physically elucidate fully the calculated effect of nematic order on our polymer rod, in section 3 we derive expressions for the tangent-tangent correlation functions of our polymer rod as well as the average end-to-end distance expected. In section 4 we present the theoretically expected structure factor for a polymer rod in a nematic solvent and reproduce the experimentally observed characteristic "bow-tie" scattering patterns. ${ }^{19-23}$ In section 5, and as one application of our model, we examine the mechanical properties of hemoglobin fibers. ${ }^{24-26}$ Under the physically reasonable assumption of the presence of a fairly weak nematic solvent, we are able to plausibly account for previously observed discrepan$\operatorname{cies}^{24,25}$ in the expected persistence lengths of hemoglo-

* Author for correspondence. E-mail D.R.Daniels@warwick.ac.uk. bin fibers of differing lengths. As another biological application, we model the effects of a strong nematic environment of fd virus on the elastic and conformational properties of wormlike micelles. ${ }^{18}$

\section{Theory}

The total free energy describing our combined polymer and nematic system, $F_{\text {total }}$, is given by the sum of three contributions:

$$
F_{\text {total }}=F_{n}+F_{t}+F_{\Lambda}
$$

The first contribution, $F_{n}$, is given by the usual Frank elasticity term ${ }^{1,2}$ in the one constant approximation:

$$
\begin{aligned}
F_{n}=\frac{\kappa_{n}}{2} \int \mathrm{d}^{3} x\left((\nabla \cdot \mathbf{n}(x))^{2}+(\nabla\right. & \left.\times \mathbf{n}(x))^{2}\right)+ \\
& \quad i \int \mathrm{d}^{3} x \alpha(x)\left(\mathbf{n}^{2}(x)-1\right)
\end{aligned}
$$

where $\mathbf{n}(x)$ is the nematic director vector field (see Figure 1).We have also included a local auxiliary field, $\alpha(x)$, so as to maintain the constraint $\mathbf{n}^{2}(x)=1$. The second term, $F_{t}$, is given by the usual elastic contribution of a semiflexible polymer chain: ${ }^{3}$

$$
F_{t}=\frac{\kappa_{t}}{2} \int_{0}^{L} \mathrm{~d} s\left(\frac{\partial \mathbf{t}(s)}{\partial s}\right)^{2}+i \int_{0}^{L} \mathrm{~d} s \beta(s)\left(\mathbf{t}^{2}(s)-1\right)
$$

where $\mathbf{t}(s)=\partial \mathbf{x}(s) / \partial s$ is the polymer rod tangent vector (see Figure 1). We have again included an additional local auxiliary field, $\beta(s)$, to enforce the constraint $\mathbf{t}^{2}(s)$ $=1$. The third component of the total free energy, $F_{\Lambda}$, represents the interaction of the nematic environment with the polymer backbone and is given by

$$
F_{\Lambda}=i \int_{0}^{L} \mathrm{~d} s \boldsymbol{\Lambda}(s) \cdot(\mathbf{n}(\mathbf{x}(s))-\mathbf{t}(s))+\frac{1}{2 \Gamma} \int_{0}^{L} \mathrm{~d} s \Lambda^{2}(s)
$$

where the value of the director field on the rod itself is given by $\mathbf{n}(\mathbf{x}(s))$, with $\mathbf{x}(s)$ being the position vector of the rod (see Figure 1). The nematic/polymer coupling 


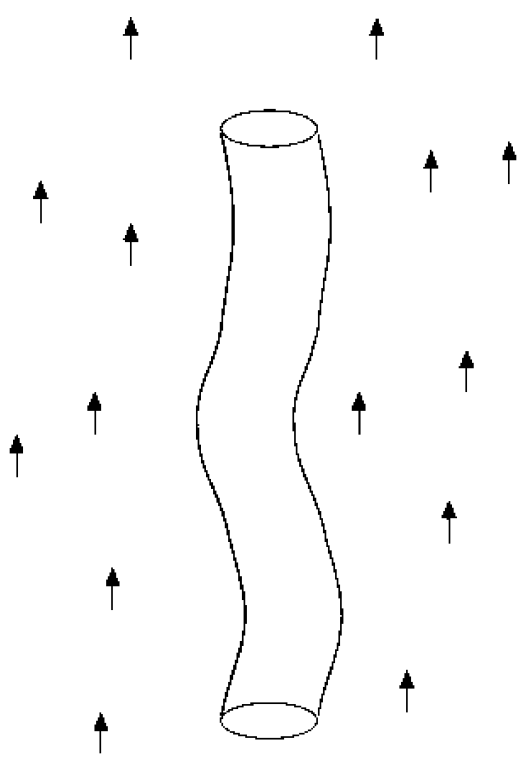

Figure 1. Schematic diagram of a polymer rod (with finite but small radius $r$ ) in a nematic solvent. The nematic director field $\mathbf{n}(x)$ is denoted by the arrows, while the tangent vector $\mathbf{t}(s)$ lies along the long axis of the rod. The value of the director field on the rod itself is given by $\mathbf{n}(\mathbf{x}(s))$, where $\mathbf{x}(s)$ is the position vector of the rod.

present in $F_{\Lambda}$ is achieved via the use of a vectorial auxiliary field, $\boldsymbol{\Lambda}(s)$, which enforces a harmonic potential (of strength $\Gamma$ ) for the polymer tangent vector, such that it prefers to point in the same direction as the nematic director field. Note that the limit $\Gamma \rightarrow \infty$ corresponds to the case of perfect coupling (or anchoring) of the nematic director to the polymer tangent vector, at the polymer's surface.

We can considerably simplify matters if we make the following parametrizations:

$$
\begin{gathered}
\mathbf{n}=\mathbf{n}_{\perp}+\sqrt{1-\mathbf{n}_{\perp}{ }^{2}} \hat{\mathbf{k}} \\
\mathbf{t}=\mathbf{t}_{\perp}+\sqrt{1-\mathbf{t}_{\perp}{ }^{2}} \hat{\mathbf{k}}
\end{gathered}
$$

and concomitantly expand both vectors, $\mathbf{t}_{\perp}$ and $\mathbf{n}_{\perp}$, to quadratic order in $F_{\text {total }}$. This procedure necessarily represents a small deformation perturbation expansion of both $\mathbf{t}$ around a relatively rigid rod (with long axis $\simeq$ $\hat{\mathbf{k}}$ ) and $\mathbf{n}$ around a relatively well-aligned nematic (with preferred axis $\simeq \hat{\mathbf{k}}$ ). We are then therefore led to consider only the fluctuations in our nematic/rod system that are perpendicular $(\perp)$ to the $\hat{\mathbf{k}}$ axis, which are governed by the following free energy, $F_{\perp}$ :

$$
\begin{gathered}
F_{\perp}=\frac{\kappa_{n}}{2} \int \mathrm{d}^{3} x\left(\left(\nabla \cdot \mathbf{n}_{\perp}\right)^{2}+\left(\nabla \times \mathbf{n}_{\perp}\right)^{2}\right)+\frac{\kappa_{t}}{2} \int_{0}^{L} \mathrm{~d} s\left(\frac{\partial \mathbf{t}_{\perp}}{\partial s}\right)^{2}+ \\
i \int_{0}^{L} \mathrm{~d} s \mathbf{\Lambda}_{\perp}(s) \cdot\left(\mathbf{n}_{\perp}(\mathbf{x}(s))-\mathbf{t}_{\perp}(s)\right)+\frac{1}{2 \Gamma} \int_{0}^{L} \mathrm{~d} s \boldsymbol{\Lambda}_{\perp}^{2}(s)
\end{gathered}
$$

Note that one great convenience of this approach, using the above parametrizations, is that it removes any dependence of $F_{\perp}$ on our auxiliary fields $\alpha(x)$ and $\beta(s)$. We are now in such a position that we can integrate out the effects of the nematic field, given by $\mathbf{n}_{\perp}$, so as to obtain the effective free energy $F_{\perp}^{\prime}$ :

$$
\begin{aligned}
F_{\perp}^{\prime}= & \frac{\kappa_{t}}{2} \int_{0}^{L} \mathrm{~d} s\left(\frac{\partial \mathbf{t}_{\perp}}{\partial s}\right)^{2}-i \int_{0}^{L} \mathrm{~d} s \boldsymbol{\Lambda}_{\perp}(s) \cdot \mathbf{t}_{\perp}(s) \\
+ & \frac{1}{2 \kappa_{n}} \int_{0}^{L} \mathrm{~d} s \int_{0}^{L} \mathrm{~d} s^{\prime} \boldsymbol{\Lambda}_{\perp}(s) \cdot \mathbf{\Lambda}_{\perp}\left(s^{\prime}\right) \times \\
& \quad \int \frac{d^{3} q}{(2 \pi)^{3}} \frac{1}{\mathbf{q}^{2}} \exp \left(i \mathbf{q} \cdot\left(\mathbf{x}(s)-\mathbf{x}\left(s^{\prime}\right)\right)\right) \\
+ & \frac{1}{2 \Gamma} \int_{0}^{L} \mathrm{~d} s \mathbf{\Lambda}_{\perp}^{2}(s)
\end{aligned}
$$

In obtaining eq 7 , we have utilized the appropriate Fourier transform of $\mathbf{n}_{\perp}$. Before we can eliminate $\Lambda_{\perp}$, we need to make sense of the three-dimensional q-space Fourier integral present in $F^{\prime}{ }_{\perp}$. To this end we again make use of a relatively rigid-rod approximation (with long axis $\simeq \hat{\mathbf{k}}$ ) and using cylindrical coordinates as follows:

$$
\mathbf{q} \cdot\left(\mathbf{x}(s)-\mathbf{x}\left(s^{\prime}\right)\right) \simeq q_{z}\left(s-s^{\prime}\right)+q_{\perp} r \cos (\Delta \varphi)
$$

where $r$ is the radius of our polymer rod and acts as a short-distance cutoff for perpendicular nematic field fluctuations. $\Delta \varphi$ is the difference in cylindrical polar angle between the vectors $\mathbf{q}$ and $\mathbf{x}$. If we also adopt the large length limit approximation (i.e., $L \rightarrow \infty$ ) and now go over to Fourier space for the polymer chain also, then we arrive at the following expression for $F_{\perp}^{\prime}$ :

$$
\begin{aligned}
F_{\perp}^{\prime} & \simeq \frac{\kappa_{t}}{2} \int_{-\infty}^{\infty} \frac{\mathrm{d} p}{2 \pi} p^{2} \mathbf{t}_{\perp}(p) \cdot \mathbf{t}_{\perp}(-p) \\
& -i \int_{-\infty}^{\infty} \frac{\mathrm{d} p}{2 \pi} \boldsymbol{\Lambda}_{\perp}(p) \cdot \mathbf{t}_{\perp}(-p) \\
& +\frac{1}{2} \int_{-\infty}^{\infty} \frac{\mathrm{d} p}{2 \pi} \boldsymbol{\Lambda}_{\perp}(p) \cdot \boldsymbol{\Lambda}_{\perp}(-p)\left(\frac{K_{0}(|p| r)}{2 \pi \kappa_{n}}+\frac{1}{\Gamma}\right)
\end{aligned}
$$

where $K_{0}(|p| r)$ is the modified Bessel function of zeroth order. Note that in arriving at eq 9 we have implicitly assumed that we can safely ignore any polymer rod "end effects" that may arise due to the nematic solvent. We will see that this assumption can be justified a posteriori, as the effect of the nematic solvent steadily decreases with decreasing polymer rod length.

We now eliminate $\Lambda_{\perp}(p)$ from $F^{\prime}{ }_{\perp}$, leaving us with our final, effective free energy, $F\left[\mathbf{t}_{\perp}\right]$, that describes small fluctuations of the component of the polymer rod tangent vector, $\mathbf{t}_{\perp}(p)$, perpendicular to the polymer rod axis (given by $\simeq \hat{\mathbf{k}}$ ):

$$
\begin{aligned}
F\left[\mathbf{t}_{\perp}\right]=\frac{\kappa_{t}}{2} \int_{-\infty}^{\infty} \frac{\mathrm{d} p}{2 \pi} p^{2} \mathbf{t}_{\perp}(p) \cdot \mathbf{t}_{\perp}(-p)+ \\
\\
\quad \frac{1}{2} \int_{-\infty}^{\infty} \frac{\mathrm{d} p}{2 \pi}\left(\frac{K_{0}(|p| r)}{2 \pi \kappa_{n}}+\frac{1}{\Gamma}\right)^{-1} \mathbf{t}_{\perp}(p) \cdot \mathbf{t}_{\perp}(-p)
\end{aligned}
$$

The above free energy, $F\left[\mathbf{t}_{\perp}\right]$, can be viewed as constituting a "microscopic" model derivation of similar, more phenomenologically inspired models of the effects of a nematic solvent on a polymer rod. ${ }^{13-18}$ Furthermore, the functional form and strength of the coupling to the nematic environment of a polymer rod are given, using the model outlined in this work and derived above, from "first principles" with a minimum of phenomenological (albeit well motivated) terms or coupling constants appearing in the final free energy.

\section{Correlations}

With $F\left[\mathbf{t}_{\perp}\right]$ now in our possession, we can, in principle, calculate all of the properties of interest of a polymer rod in a nematic solvent, for example correlation func- 
tions. The perpendicular tangent-tangent correlator (for Fourier modes $p$ ) is given by

$$
\left\langle\mathbf{t}_{\perp}(p) \cdot \mathbf{t}_{\perp}(-p)\right\rangle=\frac{2}{\kappa_{t}}\left(p^{2}+\frac{2 \pi \kappa_{n}}{\kappa_{t}} \frac{1}{K_{0}(|p| r)+\frac{2 \pi \kappa_{n}}{\Gamma}}\right)^{-1}
$$

As $|p| r \rightarrow 0$ (using $K_{0}(|p| r) \simeq-\ln (|p| r)$ ), note the logarithmic dependence on wave-mode $p$ present in the second term of eq 11. Such similar, and closely related, logarithmic behavior (termed "super" or "hyper" diffusion $^{17}$ ) was also found to be present in ref 16 when dealing with directed polymers, in ref 18 when considering semiflexible polymers in a nematic phase of rodlike virus, and also occurs frequently when considering the electrostatic interactions of polymer chains (see e.g. ref 27 for further details). From eq 11 we can clearly see that on short length scales (high $p$ ) tangent-tangent correlations are mainly bending stiffness dominated, whereas for large length scales (low $p$ ), the correlations of the tangent vector become more and more strongly influenced by the effect of the nematic solvent. Also, from eq 11, we can explicitly see the appearance of an important characteristic length scale for our system, $l$ $\sim \sqrt{\kappa_{t} / \kappa_{n}}$, which roughly demarcates the crossover between polymer stiffness $\left(\kappa_{t}\right)$ and nematic $\left(\kappa_{n}\right)$ dominated behavior.

The perpendicular tangent vector correlation function $\left\langle\mathbf{t}_{\perp}(s) \cdot \mathbf{t}_{\perp}\left(s^{\prime}\right)\right\rangle$ is given as usual (via Fourier transform) by

$$
\left\langle\mathbf{t}_{\perp}(s) \cdot \mathbf{t}_{\perp}\left(s^{\prime}\right)\right\rangle=\int_{-\infty}^{\infty} \frac{\mathrm{d} p}{2 \pi} \exp \left(i p\left(s-s^{\prime}\right)\right)\left\langle\mathbf{t}_{\perp}(p) \cdot \mathbf{t}_{\perp}(-p)\right\rangle
$$

Furthermore, using eq 12 , we can now estimate the full tangent-tangent correlator, $\left\langle\mathbf{t}(s) \cdot \mathbf{t}\left(s^{\prime}\right)\right\rangle$, as follows. Recalling our ansatz for $\mathbf{t}(s)$ from eq 5 , we can approximately write

$$
\begin{gathered}
\left\langle\mathbf{t}(s) \cdot \mathbf{t}\left(s^{\prime}\right)\right\rangle \simeq 1-\frac{1}{2}\left\langle\left(\mathbf{t}_{\perp}(s)-\mathbf{t}_{\perp}\left(s^{\prime}\right)\right)^{2}\right\rangle \\
\simeq \exp \left(-\frac{1}{2}\left\langle\left(\mathbf{t}_{\perp}(s)-\mathbf{t}_{\perp}\left(s^{\prime}\right)\right)^{2}\right\rangle\right)
\end{gathered}
$$

As a useful consistency check, eq 13 also satisfies the appropriate condition of $\left\langle\mathbf{t}^{2}(s)\right\rangle=1$ for all $s$. We can also now write down the approximate expression for the expectation value of the full end-to-end vector via

$$
\left\langle(\mathbf{x}(L)-\mathbf{x}(0))^{2}\right\rangle \simeq \int_{0}^{L} \mathrm{~d} s \int_{0}^{L} \mathrm{~d} s^{\prime}\left\langle\mathbf{t}(s) \cdot \mathbf{t}\left(s^{\prime}\right)\right\rangle
$$

To get a better physical insight into the above correlation functions, it is an illuminating exercise to consider the following limits of interest:

3.1. Intrinsic Bending Stiffness Dominated. This regime is given by $\left\{\Gamma, \kappa_{n}\right\} \rightarrow 0$, such that the effects of the nematic environment are completely absent, and we recover the typical behavior of a familiar semiflexible chain. ${ }^{3}$ In this limit, we get from above the following results:

$$
\begin{gathered}
\left\langle\mathbf{t}_{\perp}(p) \cdot \mathbf{t}_{\perp}(-p)\right\rangle \simeq \frac{2}{\kappa_{t} p^{2}} \\
\left\langle\mathbf{t}_{\perp}(s) \cdot \mathbf{t}_{\perp}\left(s^{\prime}\right)\right\rangle \simeq \frac{2 L}{\pi^{2} \kappa_{t}}-\frac{\left|s-s^{\prime}\right|}{\kappa_{t}} \\
\left\langle\left(\mathbf{t}_{\perp}(s)-\mathbf{t}_{\perp}\left(s^{\prime}\right)\right)^{2}\right\rangle \simeq 2 \frac{\left|s-s^{\prime}\right|}{\kappa_{t}} \\
\left\langle\mathbf{t}(s) \cdot \mathbf{t}\left(s^{\prime}\right)\right\rangle \simeq \exp \left(-\frac{\left|s-s^{\prime}\right|}{\kappa_{t}}\right)
\end{gathered}
$$

As a nice consistency check, note from eq 15 that we are able to recover (using our approximations) the canonical behavior ${ }^{3}$ of a semiflexible chain (in the absence of a nematic): namely, that the full tangent vector correlator, $\left\langle\mathbf{t}(s) \cdot \mathbf{t}\left(s^{\prime}\right)\right\rangle$, decays exponentially with a characteristic persistence length given by $^{3} l_{p} \sim \kappa_{t}$.

3.2. Nematic Solvent Dominated. This regime is given by $\left\{\Gamma, \kappa_{n}\right\} \rightarrow \infty$, such that the effects of the nematic environment are completely dominant. In this limit, we get from above the following results:

$$
\begin{gathered}
\left\langle\mathbf{t}_{\perp}(p) \cdot \mathbf{t}_{\perp}(-p)\right\rangle \simeq \frac{1}{\pi \kappa_{n}} K_{0}(|p| r) \\
\left\langle\mathbf{t}_{\perp}(s) \cdot \mathbf{t}_{\perp}\left(s^{\prime}\right)\right\rangle \simeq \frac{1}{2 \pi \kappa_{n}} \frac{1}{\sqrt{r^{2}+\left(s-s^{\prime}\right)^{2}}} \\
\left\langle\left(\mathbf{t}_{\perp}(s)-\mathbf{t}_{\perp}\left(s^{\prime}\right)\right)^{2}\right\rangle \simeq \frac{1}{\pi \kappa_{n}}\left(\frac{1}{r}-\frac{1}{\sqrt{r^{2}+\left(s-s^{\prime}\right)^{2}}}\right) \\
\left\langle\mathbf{t}(s) \cdot \mathbf{t}\left(s^{\prime}\right)\right\rangle \simeq \exp \left(-\frac{1}{2 \pi \kappa_{n}}\left(\frac{1}{r}-\frac{1}{\sqrt{r^{2}+\left(s-s^{\prime}\right)^{2}}}\right)\right)
\end{gathered}
$$

Note from eq 16 that now, in the presence of a strong nematic solvent, the full tangent vector correlator, $\left\langle\mathbf{t}(s) \cdot \mathbf{t}\left(s^{\prime}\right)\right\rangle$, decays as a power law $\sim\left(1 /\left|s-s^{\prime}\right|\right)$ for large $\left(s-s^{\prime}\right)$. This power law decay behavior means that in the nematic dominated phase tangent vector correlations (as given by eq 16) typically decay much more slowly than the familiar exponential decay behavior found for tangent vector correlations in the intrinsic bending stiffness dominated phase (as given by eq 15).

\section{Scattering}

In this section we examine the typical scattering behavior expected for an isolated polymer rod in a nematic environment, utilizing the theory detailed above. We define the structure factor of our polymer rod as follows:

$$
\begin{gathered}
S(q)=\langle\rho(q) \rho(-q)\rangle \\
=\frac{1}{L^{2}} \int_{0}^{L} \mathrm{~d} s \int_{0}^{L} \mathrm{~d} s^{\prime}\left\langle\exp \left(i \mathbf{q} \cdot\left(\mathbf{x}(s)-\mathbf{x}\left(s^{\prime}\right)\right)\right\rangle\right.
\end{gathered}
$$

where $\rho(q)=(1 / L) \int_{0}^{L} \mathrm{~d} s \exp (i \mathbf{q} \cdot \mathbf{x}(s))$ is the Fourier transform of the polymer density. We now approximate

$$
\begin{gathered}
\mathbf{q} \cdot\left(\mathbf{x}(s)-\mathbf{x}\left(s^{\prime}\right)\right) \simeq q_{z}\left(s-s^{\prime}\right)+\int_{s^{\prime}}^{s} \mathrm{~d} u \mathbf{q}_{\perp} \cdot \mathbf{t}_{\perp}(u) \\
\simeq q_{z}\left(s-s^{\prime}\right)+\mathbf{q}_{\perp} \cdot\left(\mathbf{x}_{\perp}(s)-\mathbf{x}_{\perp}\left(s^{\prime}\right)\right)
\end{gathered}
$$

such that we can now average eq 17 over $\mathbf{t}_{\perp}(s)$, using eq 12 , to obtain 


$$
\begin{aligned}
& S\left(q_{z}, q_{\perp}\right) \simeq \frac{1}{L^{2}} \int_{0}^{L} \mathrm{~d} s \int_{0}^{L} \mathrm{~d} s^{\prime} \exp \left(i q_{z}\left(s-s^{\prime}\right)-\right. \\
&\left.\frac{q_{\perp}^{2}}{4}\left\langle\left(\mathbf{x}_{\perp}(s)-\mathbf{x}_{\perp}\left(s^{\prime}\right)\right)^{2}\right\rangle\right)
\end{aligned}
$$

where

$$
\begin{aligned}
\left\langle\left(\mathbf{x}_{\perp}(s)-\right.\right. & \left.\left.\mathbf{x}_{\perp}\left(s^{\prime}\right)\right)^{2}\right\rangle= \\
& \int_{0}^{\infty} \frac{\mathrm{d} p}{\pi}\left\langle\mathbf{t}_{\perp}(p) \cdot \mathbf{t}_{\perp}(-p)\right\rangle \frac{2}{p^{2}}\left(1-\cos p\left(s-s^{\prime}\right)\right)
\end{aligned}
$$

$S\left(q_{z}, q_{\perp}\right)$ depends on both $q_{z}$ and $q_{\perp}$, and in what follows it proves convenient to evaluate eq 19 numerically. Shown in Figures $2-4$ are plots of $S\left(Q_{z}, Q_{\perp}\right)$ (where $Q_{z}$ $=q_{z} L$ and $\left.Q_{\perp}=q_{\perp} L\right)$ for three different values of the Frank elasticity constant, with all other constant parameters held fixed. We can see from Figures $2-4$ the characteristic "bow-tie" scattering pattern as typically found for nematic rods. ${ }^{19-23}$ We can also see from Figures 2-4 that as the Frank constant increases (i.e., the solvent/environment becomes more nematic), the associated polymer rod scattering becomes less intense along the $Q_{z}$ axis. This is consistent with the physical picture that as the nematic order increases, the polymer rod becomes more and more aligned along the nematic director. ${ }^{19-23}$

\section{Applications to Biological Polymers}

We now apply our theory to two cases of biological interest, which can be said to be representative of two consistent limits of our model. Our model assumes from almost the outset a small deformation expansion for polymer conformations, around a relatively straight rod. There are two limits in which such a description remains valid. One is when the semiflexible polymer possesses a high bending modulus (or persistence length) and is intrinsically stiff to begin with, such that the effects of the nematic environment can be taken to be a small perturbation of the (already) rigid-rod behavior. This limit will be considered in section 5.1. The other limit is when the polymer is intrinsically floppy and inherently more random coil like, such that the nematic solvent must now completely dominate its conformational properties to produce the concomitant and necessary type of rigid-rod behavior expected. This case will be considered in section 5.2.

5.1. Hemoglobin Fibers (Intrinsic Bending Stiffness Dominated). We now apply our theory for a polymer rod in a nematic solvent to the biologically interesting case of the mechanical properties of single hemoglobin fibers. ${ }^{24-26}$ Such long, slender, and relatively stiff hemoglobin fibers are responsible for diseases such as sickle cell anaemia, and it is therefore important that we are able to characterize their elastic properties accurately. To this end, following refs 24 and 25, we focus on just one of the transverse $(\perp)$ displacement vectors, $x(s)$, which we write in terms of discrete Fourier modes as $x(s)=\sum_{p=1}^{\infty} x_{p} \sin (p \pi s / L)$. From eq 11 we can write down the expression for $\left\langle x_{p}{ }^{2}\right\rangle$ immediately as

$$
\left\langle x_{p}{ }^{2}\right\rangle=\frac{2 L^{3}}{\pi^{4} \kappa_{t}} \frac{1}{p^{2}}\left(p^{2}+\frac{2 \kappa_{n} L^{2}}{\pi \kappa_{t}} \frac{1}{K_{0}\left(|p| \frac{\pi r}{L}\right)+\frac{2 \pi \kappa_{n}}{\Gamma}}\right)^{-1}
$$

from which we can also simply write down the expres-

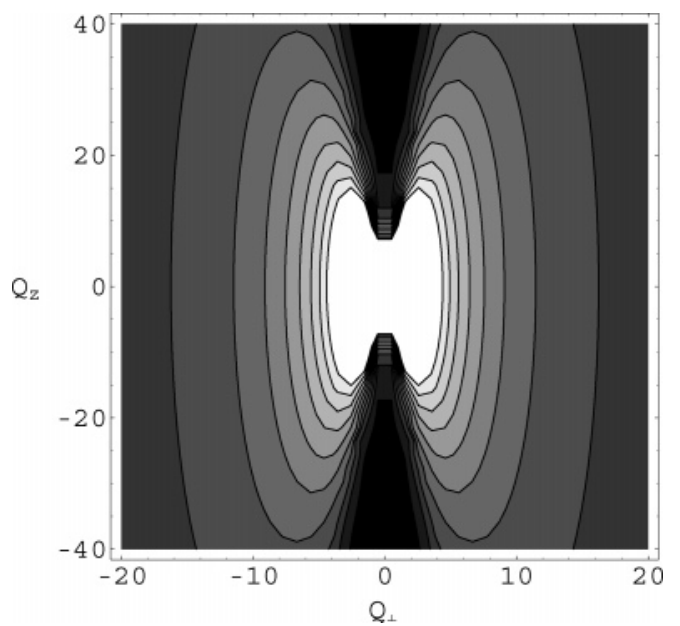

Figure 2. Isointensity contour plot of $S\left(Q_{z}, Q_{\perp}\right)$ vs $\left(Q_{\perp}, Q_{z}\right)$ with $\kappa_{t} / L=10^{-1}, L / r=10^{2}, \Gamma L=10^{4}$, and a value of the Frank elasticity constant given by $\kappa_{n} L=3 \times 10^{-3}$.

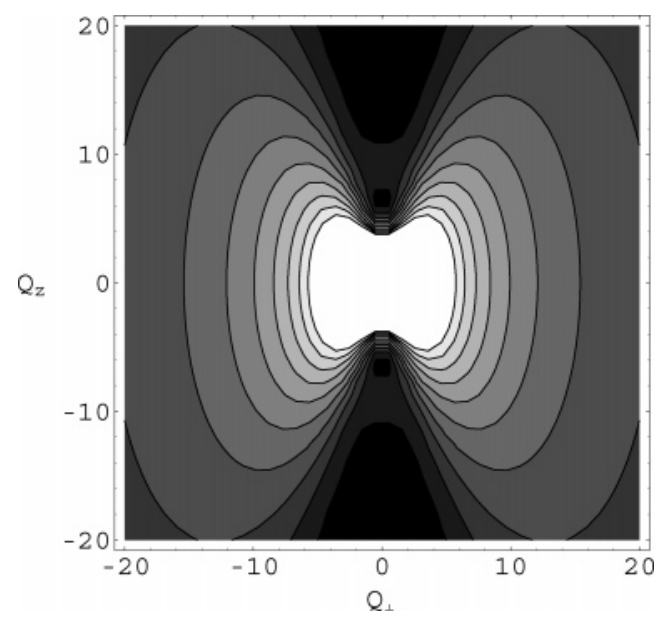

Figure 3. Isointensity contour plot of $S\left(Q_{z}, Q_{\perp}\right)$ vs $\left(Q_{\perp}, Q_{z}\right)$ with $\kappa_{t} / L=10^{-1}, L / r=10^{2}, \Gamma L=10^{4}$, and a value of the Frank elasticity constant given by $\kappa_{n} L=3 \times 10^{-1}$.

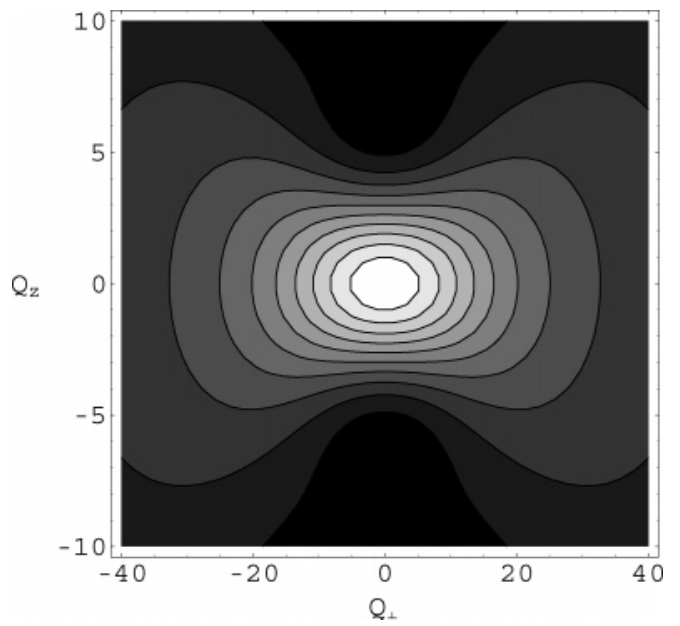

Figure 4. Isointensity contour plot of $S\left(Q_{z}, Q_{\perp}\right)$ vs $\left(Q_{\perp}, Q_{z}\right)$ with $\kappa_{\mathrm{t}} / L=10^{-1}, L / r=10^{2}, \Gamma L=10^{4}$, and a value of the Frank elasticity constant given by $\kappa_{n} L=3 \times 10^{1}$.

sion for the mean-squared amplitude at the midpoint, $\left\langle x^{2}(L / 2)\right\rangle$, as

$$
\left\langle x^{2}(L / 2)\right\rangle=\sum_{p=1}^{\infty}\left\langle x_{2 p-1}{ }^{2}\right\rangle
$$


Table 1. Experimental Data for Average Hemoglobin Fiber Midpoint Deviations (Taken from Refs 24 and 25)

\begin{tabular}{cccc} 
& $\left\langle x^{2}(L / 2)\right\rangle\left(\mu \mathrm{m}^{2}\right)$ & $L(\mu \mathrm{m})$ & $r(\mathrm{~nm})$ \\
\hline fiber 1 & 0.0868 & 10 & 10 \\
fiber 2 & 0.0002 & 1 & 10
\end{tabular}

Experimental data on single hemoglobin fibers taken from refs 24 and 25 are shown in Table 1 . Values given for $\left\langle x^{2}(L / 2)\right\rangle$ represent the best-fit values obtained after averaging over many individual fibers (see refs 24 and 25 for further details). If one postulates the presence of a weak nematic solvent, we can now straightforwardly use eq 22 and the experimental data in Table 1 to find $\kappa_{t}$ and $\kappa_{n}$. Numerically, we find the best fit values of $\kappa_{t}$ $=102 k_{\mathrm{B}} T \mu \mathrm{m}$ and $\kappa_{n}=13 k_{\mathrm{B}} T \mu \mathrm{m}^{-1}$ for both fibers, in the limit of perfect anchoring of the nematic to the fiber surface (i.e., $\Gamma \rightarrow \infty$ ). The single value of $\kappa_{t}$ obtained in this work (where the effects of an assumed weak nematic solvent are taken into account) is to be compared with those obtained in refs 24 and 25 where the possible effects of a nematic solvent were not taken into account. Reference 24 and 25 find values of $\kappa_{t}=240 k_{\mathrm{B}} T$ $\mu \mathrm{m}$ for the longer fiber and $\kappa_{t}=130 k_{\mathrm{B}} T \mu \mathrm{m}$ for the shorter fiber. A possible explanation for this discrepancy in $\kappa t$ values between the work presented here and refs 24 and 25 is as follows. Without including the effects of a postulated nematic solvent, refs 24 and 25 do a pretty good job of predicting $\kappa_{t}$ for the shorter fiber but substantially overestimate $\kappa_{t}$ for the longer fiber. Without including the effects of any posited nematic solvent, refs 24 and 25 must account for the experimentally observed midpoint deviations in the longer fiber via an overcompensatingly high and stiff value for $\kappa_{t}$. Whereas in the work presented here, the intrinsic stiffness of the polymer $\operatorname{rod}\left(\kappa_{t}\right)$ remains the same, and we can plausibly account for the experimentally observed midpoint deviations via assuming the presence of a relatively weak nematic solvent $\left(\kappa_{n}\right)$. Furthermore, as shown by comparing this work and that of refs 24 and 25, the "stiffening" effects of an assumed nematic solvent increase as the length of the polymer rod increases, which also agrees with what one would intuitively expect physically. ${ }^{16-18}$

Assuming that if the solution surrounding the fiber were to be shown experimentally to be weakly nematic, then it is perhaps not surprising that the value of the Frank constant $\left(\kappa_{n}\right)$ required in this work is roughly $1 / 200$ that of typical values to be found in conventional synthetic liquid crystals. After all, the typical size of a nematic rod to be found in synthetic liquid crystals is a few orders of magnitude smaller than the scale of the fibers of interest in this work. Furthermore, via the relation ${ }^{3} \kappa_{n} \sim k_{\mathrm{B}} T / d$, the appropriate length scale implied by our value of $\kappa_{n}=13 k_{\mathrm{B}} T \mu \mathrm{m}^{-1}$ is around $d \sim$ $80 \mathrm{~nm}$, which is consistently and reassuringly at least of the same order of magnitude as the diameter of our polymer rods. Interestingly, comparable values of $\kappa_{n}$ to that used in the work presented here have recently also been found in the nematic phase of rodlike virus. ${ }^{18}$

5.2. Wormlike Micelles and fd Virus (Nematic Solvent Dominated). Recently ${ }^{18}$ fluorescence microscopy has been used to experimentally study the tangenttangent correlations of wormlike micelles immersed in a nematic solvent of rodlike-shaped fd virus (see ref 18 for further details) at varying fd concentrations. There it is found experimentally that in the nematic phase of the fd virus the wormlike micelles adopt a highly elongated, straight, rodlike conformation, whereas in

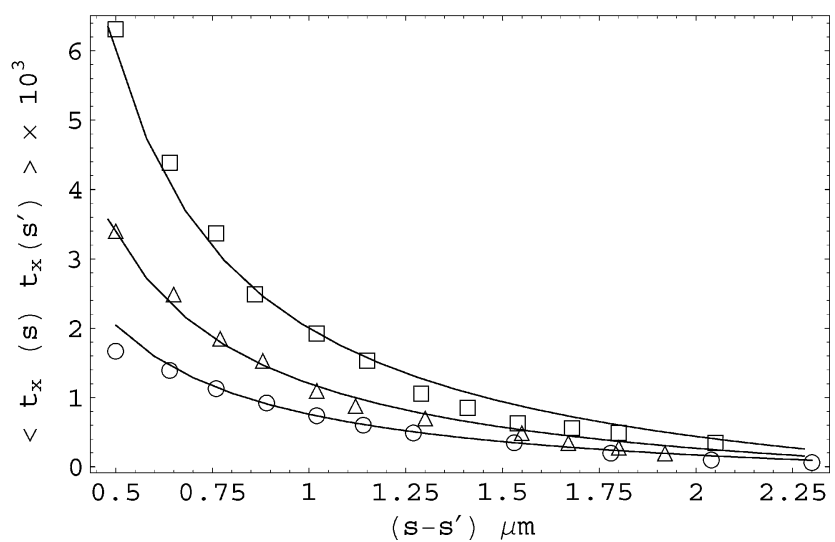

Figure 5. Model best fits of $\left\langle t_{x}(s) t_{x}\left(s^{\prime}\right)\right\rangle$ vs $\left(s-s^{\prime}\right)$ for wormlike micelles in a nematic solvent of fd virus. The concentrations of fd virus are given by $c_{\mathrm{fd}}=39 \mathrm{mg} / \mathrm{mL}$ (open squares), $c_{\mathrm{fd}}=$ $51 \mathrm{mg} / \mathrm{mL}$ (open triangles), and $c_{\mathrm{fd}}=97 \mathrm{mg} / \mathrm{mL}$ (open circles). (Experimental data points are taken from ref 18. )

Table 2. Model Best Fits for the Frank Constant $\kappa_{n}$ of a Nematic Solvent of fd Virus, at Varying Concentrations ${ }^{a}$

\begin{tabular}{cc}
\hline$c_{\mathrm{fd}}(\mathrm{mg} / \mathrm{mL})$ & $\kappa_{n}\left(k_{\mathrm{B}} T \mu \mathrm{m}^{-1}\right)$ \\
\hline 39 & 28 \\
51 & 45 \\
97 & 70
\end{tabular}

${ }^{a}$ We assume throughout that $L=50 \mu \mathrm{m}, r=7 \mathrm{~nm}, \kappa_{t}=0.5$ $k_{\mathrm{B}} T \mu \mathrm{m}$, and $\Gamma=\infty$ (with experimental measurements taken from refs 18 and 28).

the isotropic phase of the fd virus the wormlike micelles adopt a highly compact, random coillike conformation. ${ }^{18}$

To accurately model the experimental data of ref 18 , eq 12 becomes

$$
\left\langle t_{x}(s) t_{x}\left(s^{\prime}\right)\right\rangle=\frac{1}{2} \int_{\pi / L}^{\infty} \frac{\mathrm{d} p}{\pi} \cos \left(p\left(s-s^{\prime}\right)\right)\left\langle\mathbf{t}_{\perp}(p) \cdot \mathbf{t}_{\perp}(-p)\right\rangle
$$

Note the need to incorporate finite length effects into in eq 23 via a low Fourier mode cutoff $(\pi / L)$ in order to accurately capture the experimental data. Note also the factor of $1 / 2$ that appears on the right-hand side of eq 23 , which is required since we are only dealing in this instance with the $x$ component of perpendicular tangent vector fluctuations. The proper accounting for this factor in this work (as present in eq 23) corrects an error in an analogous expression to be found in the work of ref 18.

Shown in Figure 5 are fits of our model (given by eq 23 ) to the experimental data of ref 18 . The data points are given for three different values of the concentration of nematic fd virus $\left(c_{\mathrm{fd}}\right)$. Note that the magnitude of the correlation function decreases as $c_{\mathrm{fd}}$ increases. In all the model plots shown, we have taken $L=50 \mu \mathrm{m}, r=7$ $\mathrm{nm}$, and $\kappa_{t}=0.5 k_{\mathrm{B}} T \mu \mathrm{m} \cdot{ }^{18,28}$ We have also taken $\Gamma=\infty$ for all the model fits to the experimental data (corresponding to perfect anchoring of the nematic at the polymer surface). This provides us with a stringent test of our model, in that the only remaining parameter now available to us for fitting, is the nematic Frank constant, $\kappa_{n}$, for the fd virus.

Shown in Table 2 are the best fit values for the Frank nematic constant $\kappa_{n}$, for the three different concentrations of nematic fd virus used in Figure 5 above. ${ }^{18}$ Note that as the concentration of fd virus $\left(c_{\mathrm{fd}}\right)$ increases, then $\kappa_{n}$ increases also, as one would expect intuitively. Furthermore, the best fit values of $\kappa_{n}$ given in Table 2 
are consistent with previous measurements of the Frank elasticity constants for fd virus samples prepared under similar experimental conditions. ${ }^{29}$

\section{Conclusions}

We have studied theoretically a rodlike polymer immersed in a nematic environment and described the effect of a nematic solvent on small fluctuations of the tangent vector perpendicular to the rod axis. We were thus able to calculate the physically interesting properties associated with our combined polymer and nematic system, including tangent-tangent correlation functions, and the scattering structure factor, which reproduces qualitatively known experimental results well. ${ }^{19-23}$

The work presented here can be viewed as constituting a microscopic model derivation of other, more phenomenologically inspired, theories ${ }^{13-18}$ that deal with similar polymer/nematic systems. As one biologically important application of our model, we analyzed the effects of a postulated nematic solvent on the mechanical and elastic properties of individual hemoglobin fibers. ${ }^{24-26}$ Under the physically reasonable assumption of the presence of a fairly weak nematic solvent, we are able to plausibly account for previously observed discrepancies in the apparent rigidities of hemoglobin fibers of varying lengths. ${ }^{24,25}$ As another biological application, we modeled the effects of a strong nematic environment of $\mathrm{fd}$ virus on the elastic and conformational properties of wormlike micelles. ${ }^{18} \mathrm{In}$ terestingly, both of the above applications seem to suggest that we can successfully model the experimental data by just considering the limit of perfect anchoring of the nematic to the polymer surface (i.e., $\Gamma \rightarrow \infty$ ). This (rather appealingly) further suggests that for many practical modeling purposes one can safely apply Occam's razor to $\Gamma$ (which is after all a somewhat ad hoc, and presumably extremely difficult to measure, quantity) and eliminate it as a free-fit parameter from our theory, by setting it to be very large (i.e., infinity) from the outset. Finally, we have shown in general that the presence of a nematic environment can have important and interesting effects on the conformational, elastic, and mechanical properties of single polymer chains. In light of this, the work presented here should also prove to be similarly important for other long, slender, and semiflexible biological macromolecules, such as TMV, DNA, and proteins. The possibly interesting effects of the presence of a nematic solvent on fiber self-assembly and organization are left to future work.
Acknowledgment. This work was supported by NIH Grant HL 58512 from the National Heart Lung and Blood Institute.

\section{References and Notes}

(1) de Gennes, P. G.; Prost, J. The Physics of Liquid Crystals; Oxford University Press: New York, 1995.

(2) Chaikin, P. M.; Lubensky, T. C. Principles of Condensed Matter Physics; Cambridge University Press: New York, 2000

(3) Doi, M.; Edwards, S. F. The Theory of Polymer Dynamics; Clarendon Press: Oxford, 1988.

(4) Odijk, T. J. Chem. Phys. 1996, 105, 1270.

(5) Rill, R. L.; Strzelecka, T. E.; Davidson, M. W.; Van Winkle, D. H. Physica A 1991, 176, 87.

(6) Kassapidou, K.; Heenan, R. K.; Jesse, W.; Kuil, M. E. Macromolecules 1995, 28, 3230.

(7) Hentschke, R.; Herzfeld, J. Phys. Rev. A 1991, 43, 7019.

(8) Viamontes, J.; Tang, J. X. Phys. Rev. E 2003, 67, 040701.

(9) Das, P.; Roy, J.; Chakrabarti, N.; Basu, S.; Das, U. J. Chem. Phys. 2002, 116, 9028

(10) Graf, H.; Lowen, H. Phys. Rev. E 1999, 59, 1932.

(11) Fraden, S.; Maret, G. Phys. Rev. E 1993, 48, 2816.

(12) Oldenbourg, R.; Wen, X.; Meyer, R. B.; Caspar, D. L. D. Phys. Rev. Lett. 1988, 61, 1851.

(13) Warner, M.; Gunn, J. M. F.; Baumgartner, A. B. J. Phys. A $\mathbf{1 9 8 5}, 18,3007$.

(14) Carri, G. A.; Muthukumar, M. J. Chem. Phys. 1998, 109, 11117.

(15) Kholodenko, A. L.; Vilgis, T. A. Phys. Rep. 1998, 298, 251.

(16) Kamien, R. D.; Le Doussal, P.; Nelson, D. R. Phys. Rev. A 1992, 45,8727 .

(17) de Gennes, P. G. In Polymer Liquid Crystals; Academic: New York, 1982.

(18) Dogic, Z.; Zhang, J.; Lau, A. W. C.; Aranda-Espinoza, H.; Dalhaimer, P.; Discher, D. E.; Janmey, P. A.; Kamien, R. D.; Lubensky, T. C.; Yodh, A. G. Phys. Rev. Lett. 2004, 92, 125503.

(19) Purdy, K. R.; Dogic, Z.; Fraden, S.; Ruhm, A.; Lurio, L.; Mochrie, S. G. J. Phys. Rev. E 2003, 67, 031708.

(20) Hamley, I. W.; Garnett, S.; Luckhurst, G. R.; Roskilly, S. J.; Skov Pedersen, J.; Richardson, R. M.; Seddon, J. M. J. Chem. Phys. 1996, 104, 10046.

(21) Xiaolei, X.; Wen, X.; Meyer, R. B. Physica A 1991, 176, 63

(22) Pelletier, O. Eur. Phys. J. E 2000, 2, 191.

(23) Wagner, N. J.; Walker, L. M. Macromolecules 1995, 28, 5075

(24) Turner, M. S.; Wang, J. C.; Jones, C.; Ferrone, F. A.; Briehl, R. W.; Josephs, R. Langmuir 2002, 18, 7182.

(25) Wang, J. C.; Turner, M. S.; Agarwal, G.; Kwong, S.; Josephs, R.; Ferrone, F. A.; Briehl, R. W. J. Mol. Biol. 2002, 315, 601.

(26) Hentschke, R.; Herzfeld, J. Phys. Rev. A 1991, 43, 7019.

(27) Tkachenko, A. V. xxx.lanl.gov preprint 2003, cond-mat, 0303076.

(28) Dalhaimer, P.; Bates, F. S.; Discher, D. E. Macromolecules 2003, 36, 6873

(29) Dogic, Z.; Fraden, S. Langmuir 2000, 16, 7820. 